\title{
THREe Dimensionally ORdERED MACROPOROUS BiOACTIVE GLASSES
}

\author{
David C. Bell, Kai Zhang ${ }^{\&}$, Hongwei Yan", Lorraine F. Francis ${ }^{\&}$, Andreas Stein*
}

Institute of Technology Characterization Facility, ${ }^{\&}$ Department of Chemical Engineering and Materials Science, *Department of Chemistry

The University of Minnesota, Minneapolis, MN 55455

Bioactive ceramics including glasses have been used to repair and reconstruct damaged parts of the skeletal system [1]. A three dimensionally ordered macroporous (3DOM) structure can be formed using the sol-gel technique to produce a glass that is bioactive [2]. Bioactive 3DOM materials develop a biologically active, hydroxycarbonate apatite layer on their surfaces within minutes of exposure to body fluids either in vivo or in vitro. The composition and morphology of sol-gel derived glass materials have direct impact on the design of the new generation of biomaterials for use in bone regeneration, membranes and possible drug delivery systems.

The composition, processing temperature, surface chemistry and structural morphology of the solgel bioactive glasses affect the apatite formation and growth [3]. The bioactivity is enhanced by increasing the surface pore size and developing bulk porous structure. The 3DOM sol-gel bioactive glasses were synthesized for this research using monodispersed poly- methyl methacrylate, (PMMA), spheres of different sizes (100-1000 nm), followed by a sol gel process as described in $[4,5]$. The resultant 3DOM glass is characterized using SEM as shown in figure 1 and is shown to have an fcc arrangement of pores. The preparation and microstructure of solgel bioactive glasses with 3DOM structure will be presented.

To test in vitro bioactivity the 3DOM sol-gel glass is soaked at body temperature in a simulated body fluid (SBF) to observe the apatite formation. The composition and preparation of SBF is after Abe et al. [6]. The 3DOM structure, after a sufficient soaking time, completely converts to a flake like material, which was demonstrated to be crystalline hydroxycarbonate apatite (HCA) by XRD, FTIR and TEM characterization. Figure 2, shows $\mathrm{CaO}-\mathrm{SiO}_{2}-\mathrm{P}_{2} \mathrm{O}_{5} 3 \mathrm{DOM}$ sol-gel bioactive glass after soaking in SBF for 4 days. The images show that the surface is covered with HCA. The development of HCA is faster for 3DOM sol gel bioactive glasses than for traditional sol-gel bioactive glasses [2]. Differences in bioactive behaviors are directly related to the microstructural morphology; the SBF can readily penetrate the larger macropores in the 3DOM bioactive glass compared to the mesopores in traditional sol-gel processed bioactive glasses, while minor differences in chemical composition of 3DOM glass have little effect. Pore size can be adjusted by using different sizes of PMMA spheres. Factors determining apatite growth such as pore size and ordering, which is critical for skeletal reconstruction applications, are discussed.

\section{References:}

[1] Hench L.L., J. Am. Ceram. Soc., 81, 1705-1728, 1998

[2] Yan H., Zhang K., Blanford C.F., Francis L.F., Stein A., Chem. Mat., 13, 1374-1382, 2001

[3] Pereira M.M. et al. J. Am. Ceram. Soc., 78, 2463-2468, 1995.

[4] Zhang K., Yan H., Bell D.C., Stein A., Francis L.F., Trans. of Soc. for Biomaterials, 282002

[5] Holland B.T., Blanford C.F., Stein A., Science, 281, 538-540, 1998 
[6] Abe Y., et al., Chem. Mat., 12, 1134-1141, 2000

[7] Support of the University of Minnesota, IT Characterization Facility is gratefully acknowledged.
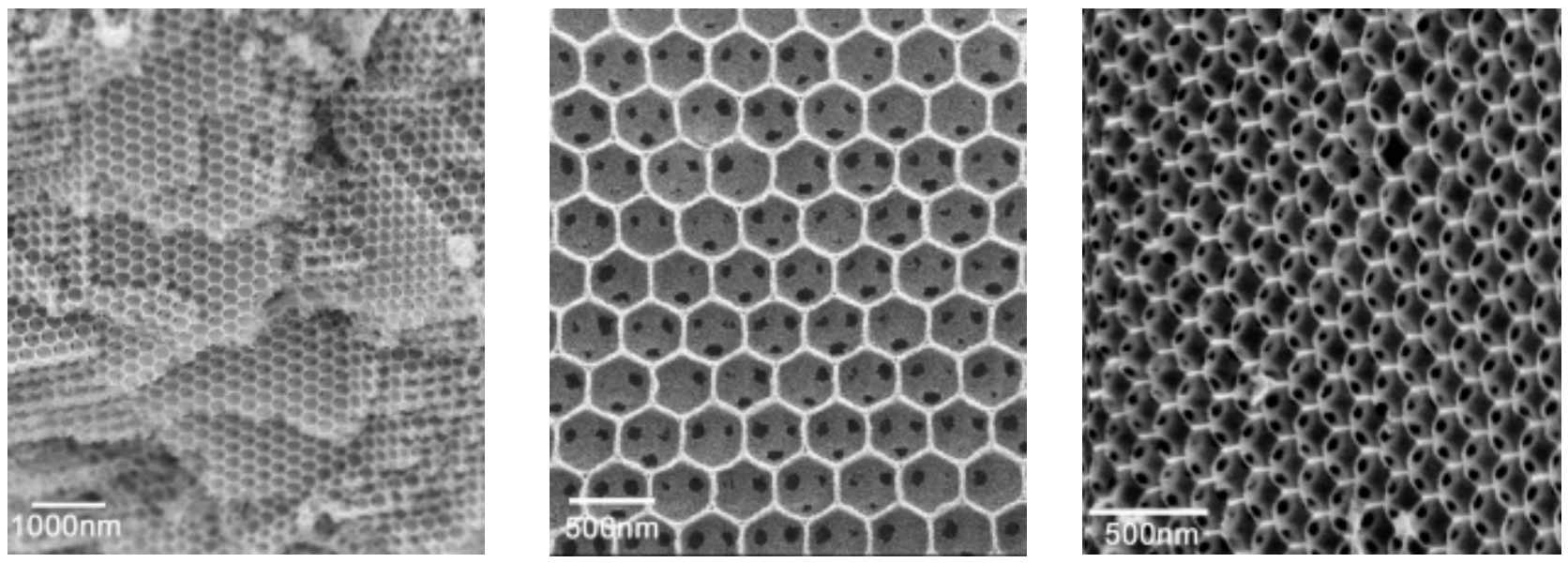

Figure 1. SEM Images of 3 DOM bioactive glass formed using the sol-gel process described, indicating the macro-sized pore structure.
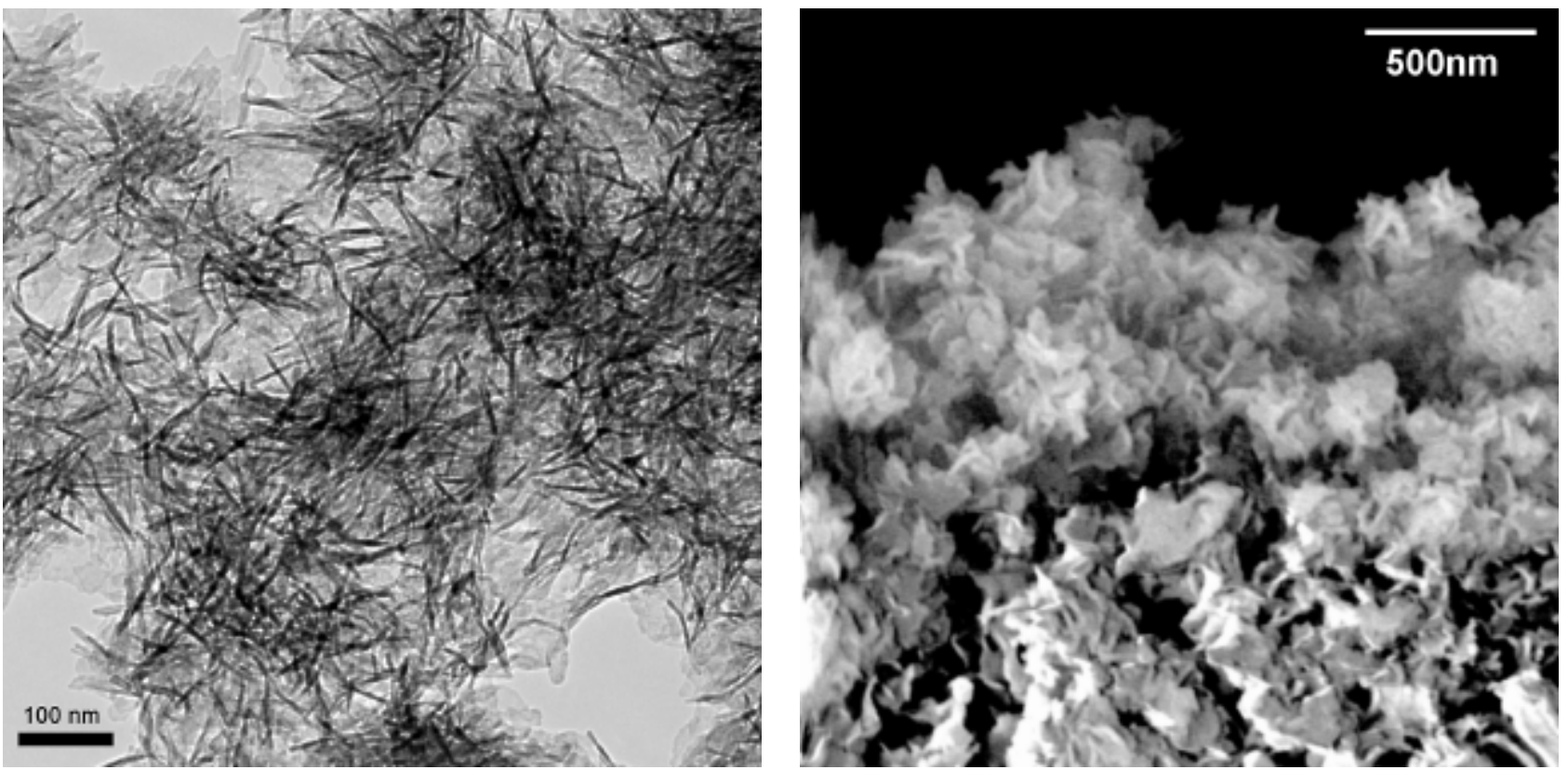

Figure 2. (Left) TEM image of apatite formation on $\mathrm{CaO}-\mathrm{SiO}_{2}-\mathrm{P}_{2} \mathrm{O}_{5} 3 \mathrm{DOM}$ sol-gel bioactive glass after soaking in SBF for 4 days. (Right) SEM image of surface of $\mathrm{CaO}-\mathrm{SiO}_{2}-\mathrm{P}_{2} \mathrm{O}_{5}$ 3DOM sol-gel bioactive glass after soaking in SBF for 4 days showing flake like HCA. 